\title{
Adherence to Treat-to-target Management in Rheumatoid Arthritis and Associated Factors: Data from the International RA BIODAM Cohort
}

\author{
Alexandre Sepriano (D), Sofia Ramiro (D), Oliver FitzGerald (D), Mikkel Østergaard (D), \\ Joanne Homik (1), Désirée van der Heijde (1), Ori Elkayam, J. Carter Thorne (i), \\ Maggie J. Larché, Gianfranco Ferraccioli, Marina Backhaus, Gerd R. Burmester (D), \\ Gilles Boire ${ }^{\circledR}$, Bernard Combe, Thierry Schaeverbeke, Alain Saraux ${ }^{(D)}$, Maxime Dougados, \\ Maurizio Rossini, Marcello Govoni (i), Luigi Sinigaglia ${ }^{(\mathbb{D})}$, Alain Cantagrel, Cheryl Barnabe (i), \\ Clifton O. Bingham III, Paul P. Tak, Dirkjan van Schaardenburg, Hilde Berner Hammer, \\ Joel Paschke, Rana Dadashova, Edna Hutchings (iD, Robert Landewé (i), \\ and Walter P. Maksymowych 1
}

ABSTRACT. Objective. Compelling evidence supports a treat-to-target (T2T) strategy for optimal outcomes in rheumatoid arthritis (RA). There is limited knowledge regarding the factors that impede implementation of T2T, particularly in a setting where adherence to T2T is protocol-specified. We aimed to assess clinical factors that associate with failure to adhere to T2T.

Methods. Patients with RA from 10 countries who were starting or changing conventional synthetic disease-modifying antirheumatic drugs and/or starting tumor necrosis factor inhibitors were followed for 2 years. Participating physicians were required per protocol to adhere to the T2T strategy. Factors influencing adherence to T2T low disease activity (T2T-LDA; 44-joint count Disease Activity Score $\leq 2.4$ ) were analyzed in 2 types of binomial generalized estimating equations models: (1) including only baseline features (baseline model); and (2) modeling variables that inherently vary over time as such (longitudinal model).

Results. A total of 571 patients were recruited and 439 (76.9\%) completed 2-year followup. Failure of adherence to T2T-LDA was noted in 1765 visits (40.5\%). In the baseline multivariable model, a high number of comorbidities (OR 1.10, 95\% CI 1.02-1.19), smoking (OR 1.32, 95\% CI 1.08-1.63) and high number of tender joints (OR $1.03,95 \%$ CI 1.02-1.04) were independently associated with failure to implement T2T, while anticitrullinated protein antibody/rheumatoid factor positivity (OR $0.63,95 \%$ CI $0.50-0.80$ ) was a significant facilitator of T2T. Results were similar in the longitudinal model.

Conclusion. Lack of adherence to T2T in the RA BIODAM cohort was evident in a substantial proportion despite being a protocol requirement, and this could be predicted by clinical features. [Rheumatoid Arthritis (RA) BIODAM cohort; ClinicalTrials.gov: NCT01476956]. (First Release February 15 2020; J Rheumatol 2020;47:809-19; doi:10.3899/jrheum.190303)

Key Indexing Terms:

RHEUMATOID ARTHRITIS BEST TREATMENT PRACTICES TREAT-TO-TARGET

From the Department of Rheumatology, Leiden University Medical Center, Leiden; Zuyderland Medical Center, Heerlen; Academic Medical Center/University of Amsterdam, Amsterdam University Medical Center, Amsterdam; Amsterdam Rheumatology and Immunology Center, locations Reade and Amsterdam, University Medical Center, Amsterdam, the Netherlands; Newman Clinical Research, Department of Rheumatology, St. Vincent's University Hospital, Dublin, Ireland; Copenhagen Center for Arthritis Research, Center for Rheumatology and Spine Diseases, Rigshospitalet - Glostrup, University of Copenhagen, Copenhagen, Denmark; University of Alberta, Edmonton, Alberta; The Arthritis Program Research Group, Newmarket, Ontario; Divisions of Rheumatology and Clinical Immunology and Allergy, McMaster University, Hamilton, Ontario; Rheumatology Department, Centre Intégré Universitaire en Santé et Services Sociaux (CIUSSS) de l'Estrie-Centre

Hospitalier Universitaire de Sherbrooke (CHUS), Université de

Sherbrooke, Sherbrooke, Quebec; Departments of Medicine and
Community Health Services, Cumming School of Medicine, University of Calgary, Calgary, Alberta; CaRE Arthritis Ltd., Edmonton, Alberta, Canada; Department of Rheumatology, Tel Aviv Sourasky Medical Center, Tel Aviv, Israel; Divisions of Rheumatology and Internal Medicine, Catholic University of the Sacred Heart, Rome; Department of Rheumatology, Università di Verona, Verona; St. Anna Hospital, Ferrara (loc. Cona); Department of Rheumatology, Istituto Ortopedico Gaetano Pini, Milan, Italy; Department of Rheumatology and Clinical Immunology, Charité University Hospital, Berlin, Germany; Departement de rhumatologie, Université de Montpellier, Centre Hospitalier Universitaire (CHU) Montpellier, Montpellier; Service de rhumatologie, Centre National de Référence des Maladies Auto-immunes Rares de l'Adulte CERAINO, and UMR1227, Lymphocytes B et Autoimmunité (LBAI), Université de Brest, INSERM, LabEx Immunothérapies Grand Ouest (IGO), Brest; Paris Descartes University, Rheumatology Department, Cochin Hospital, AP-HP, INSERM (U1153): Clinical Epidemiology and

Personal non-commercial use only. The Journal of Rheumatology Copyright @ 2020 . All rights reserved. 
Systematic reviews provide compelling evidence that treat-to-target (T2T) strategies lead to better results on any

Biostatistics, PRES Sorbonne Paris-Cité, Paris; Centre de Rhumatologie, Hôpital Pierre Paul Riquet - Purpan, CHU de Toulouse, Toulouse; Service de Rhumatologie, CHU Bordeaux Pellegrin, Bordeaux, France; Divisions of Rheumatology and Allergy and Clinical Immunology, Johns Hopkins University, Baltimore, Maryland, USA; Department of Rheumatology, Diakonhjemmet Hospital, Oslo, Norway.

This is an investigator-initiated study aimed at the clinical validation of biomarkers, which has been supported by unrestricted funding from AbbVie Corp. AbbVie had no role in the design, execution, or analysis of this study and had no role in the drafting of the manuscript.

W.P. Maksymowych is Chief Medical Officer of the International Project Management Group, CaRE Arthritis, Ltd.

A. Sepriano, MD, PhD applicant, Department of Rheumatology, Leiden University Medical Center; S. Ramiro, $M D, P h D$, Department of Rheumatology, Leiden University Medical Center, and Zuyderland Medical Center; O. FitzGerald, MD, Newman Clinical Research, Department of Rheumatology, St. Vincent's University Hospital; M. Østergaard, DMSc, Professor of Rheumatology, Copenhagen Center for Arthritis Research, Center for Rheumatology and Spine Diseases, Rigshospitalet - Glostrup, University of Copenhagen; J. Homik, FRCP $(C)$, Professor of Medicine, University of Alberta; D. van der Heijde, MD, Professor of Rheumatology, Department of Rheumatology, Leiden University Medical Center; O. Elkayam, MD, Department of Rheumatology, Tel Aviv Sourasky Medical Center; J.C. Thorne, FRCP $(C)$, The Arthritis Program Research Group; M.J. Larché, MBChB, PhD, Divisions of Rheumatology and Clinical Immunology and Allergy, McMaster University; G. Ferraccioli, MD, Professor, Divisions of Rheumatology and Internal Medicine, Catholic University of the Sacred Heart; M. Backhaus, MD, Department of Rheumatology and Clinical Immunology, Charité University Hospital; G.R. Burmester, MD, Professor of Medicine, Department of Rheumatology and Clinical Immunology, Charité University Hospital; G. Boire, FRCP $(C)$, Professor of Medicine, Rheumatology Department, CIUSSS de l'Estrie-CHUS, Université de Sherbrooke; B. Combe, MD, PhD, Professor of Rheumatology, Departement de rhumatologie, Université de Montpellier, $\mathrm{CHU}$ Montpellier; T. Schaeverbeke, MD, Professor, Service de Rhumatologie, CHU Bordeaux Pellegrin; A. Saraux, MD, Professor, Service de rhumatologie, Centre National de Référence des Maladies Auto-immunes Rares de l'Adulte CERAINO, and UMR1227, LBAI, Université de Brest, INSERM, LabEx IGO; M. Dougados, MD, Professor of Medicine, Paris Descartes University, Rheumatology Department, Cochin Hospital, AP-HP, INSERM (U1153): Clinical Epidemiology and Biostatistics, PRES Sorbonne Paris-Cité; M. Rossini, MD, Department of Rheumatology, Università di Verona; M. Govoni, MD, Associate Professor of Rheumatology, St. Anna Hospital, Ferrara (loc. Cona); L. Sinigaglia, MD, Department of Rheumatology, Istituto Ortopedico Gaetano Pini; A. Cantagrel, MD, Professor, Centre de Rhumatologie, Hôpital Pierre Paul Riquet - Purpan, CHU de Toulouse; C. Barnabe, MD, Associate Professor, Departments of Medicine and Community Health Services, Cumming School of Medicine, University of Calgary; C.O. Bingham III, $M D$, Professor, Divisions of Rheumatology and Allergy and Clinical Immunology, Johns Hopkins University; P.P. Tak, MD, Professor of Medicine, Academic Medical Center, Amsterdam University Medical Center; D. van Schaardenburg, MD, Professor of Medicine, Amsterdam Rheumatology and Immunology Center, locations Reade and Amsterdam, University Medical Center; H. Berner Hammer, MD, Department of Rheumatology, Diakonhjemmet Hospital; J. Paschke, BSc, CaRE Arthritis Ltd.; R. Dadashova, MD, CaRE Arthritis Ltd.; E. Hutchings, RN, CaRE Arthritis Ltd.; R. Landewé, MD, Academic Medical Center/University of Amsterdam, and Zuyderland Medical Center; W.P. Maksymowych, FRCP $(C)$, Professor of Medicine, University of Alberta, and Chief Medical Officer, CaRE Arthritis Ltd.

Address correspondence to Dr. W.P. Maksymowych, CaRE Arthritis, \#210, 316 Windemere Road NW, Edmonton, Alberta T6W 2Z8, Canada. E-mail: walter.maksymowych@carearthritis.com

Accepted for publication August 22, 2019. outcome that includes clinical, structural, functional, work productivity, comorbidity, and costs, irrespective of the precise characteristics of treatment and in both early and late rheumatoid arthritis (RA) ${ }^{1-7}$. This has led to the incorporation of this strategy in both American College of Rheumatology (ACR) and European League Against Rheumatism (EULAR) treatment recommendations ${ }^{8,9}$. However, lack of adherence to the T2T strategy compromises achievement of optimal outcomes and leads to disease flares ${ }^{10}$. Knowledge regarding the factors that impede implementation and adherence to $\mathrm{T} 2 \mathrm{~T}$, particularly in a setting where adherence to T2T is protocol-specified, is limited.

The Outcome Measures in Rheumatology (OMERACT) Soluble Biomarker International Working Group initiated an international, multicenter, prospective study, RA BIODAM, aimed at setting a benchmark for the design, implementation, and analysis of studies aimed at the validation of prognostic variables that are predictive of radiographic progression in RA. It was considered essential in the study design of a prospective cohort to include patients with a wide spectrum of disease activity receiving diverse treatments but adhering to a T2T strategy ${ }^{11,12}$. This would serve not only to optimize patient outcomes but also to provide an opportunity to study the relationship between change in the candidate biomarker(s) related to treatment and subsequent change in the radiographic endpoint. The RA BIODAM study therefore provided an opportunity to assess adherence to the perprotocol T2T strategy over the course of the study and to identify patient and disease characteristics that could serve as barriers and facilitators of implementation of T2T.

\section{MATERIALS AND METHODS}

Study design of RA BIODAM. This is a multicenter, multinational, prospective observational study of patients with RA and fulfilling the 2010 Rheumatoid Arthritis Classification Criteria ${ }^{13}$ recruited consecutively from rheumatologist outpatient clinics and offices in Canada $(n=9)$, the United States $(n=5)$, Israel $(n=1)$, Denmark $(n=1)$, France $(n=6)$, Germany $(n=4)$, Ireland $(n=1)$, Italy $(n=6)$, the Netherlands $(n=4)$, and Norway $(n=1)$. [Trial registration: Assess Structural Damage in Rheumatoid Arthritis Using Biomarkers and Radiography: Clinicaltrials.gov \#: NCT01476956, clinicaltrials.gov/ct2/show/NCT01476956]. The first patient was recruited on October 30,2011, and the last patient visit was May 17,2017. Details of the study design, inclusion, and exclusion criteria, have been described in the first study report ${ }^{14}$. The study recruited patients who were starting conventional synthetic disease-modifying antirheumatic drugs (csDMARD) therapy; changing csDMARD therapy, defined as an increase in dose of methotrexate (MTX) by $\geq 5 \mathrm{mg}$ weekly to a maximum dose of $25 \mathrm{mg}$ weekly, add-on of an alternative csDMARD, or switch to an alternate csDMARD; or were adding tumor necrosis factor inhibitor (TNFi) to csDMARD therapy.

Disease activity was monitored systematically every 3 months using the 44-joint count Disease Activity Score (DAS44). Changes in csDMARD and/or TNFi therapy were to be implemented according to 2010 EULAR recommendations: a target of remission (REM; DAS44 < 1.6) for patients receiving csDMARD therapy in the setting of early disease $(<2$ yrs disease duration) and a target of low disease activity (LDA) state (DAS44 $\leq 2.4$ ) for patients receiving TNFi in the setting of established disease and prior exposure to csDMARD ${ }^{15}$. If treatment change was not implemented according to the protocol procedure, a study query was sent to the investi-

Personal non-commercial use only. The Journal of Rheumatology Copyright @ 2020 . All rights reserved. 
gator requesting the reason for not adhering to T2T. One of the following options was provided for a response: patient decision (with specification), physician decision - concern regarding adverse event, physician decision current treatment acceptable, physician decision - other (with specification), other, unspecified.

The study fulfilled Good Clinical Practice Guidelines, complied with the Declaration of Helsinki, and received ethical approval from the local ethics committees (Supplementary Data, available with the online version of this article); all patients provided written informed consent.

Statistical analysis. Because rheumatologist adherence to a T2T treatment strategy was a key protocol-specified requirement, we assessed the number of visits where this was not implemented. Failure to follow T2T according to an REM/LDA target (T2T-REM/LDA according to DAS44) was defined as (1) failure to intensify treatment if REM/LDA status had not been attained, and (2) treatment intensification if REM/LDA status had been attained.

The following predictors were assessed to identify those factors associated with failure to implement T2T: (1) clinical and socioeconomic variables, including age (yrs), sex (female/male), disease duration (yrs), country (each patient nested within country) and site type (academic/ community), number of comorbidities (continuous), education (yrs), smoking status (current smoker/not current smoker); rheumatoid factor (RF; positive/negative), anticitrullinated protein antibodies (ACPA; positive/ negative), baseline medication status (csDMARD-naive/csDMARD-experienced), number of previous DMARD (continuous), treatment with biologic DMARD/csDMARD (dummy variable with 4 levels; reference: csDMARD only), treatment with oral steroids (yes/no); Health Assessment Questionnaire (HAQ; continuous); and (2) disease activity variables: swollen joint count (SJC; 0-44), tender joint count (TJC; 0-53), erythrocyte sedimentation rate $(\mathrm{ESR} ; \mathrm{mm} / \mathrm{h}), \mathrm{CRP}(\mathrm{mg} / \mathrm{l})$, patient's global assessment (0-10), physician's global assessment (0-10).

We used 2-level binomial generalized estimating equation models to test associations between potential predictors and failure to implement T2T-LDA (the primary outcome) and T2T-REM. Patients with at least 2 timepoints with available data were included in our models. Two types of models were created: (A) baseline model (only with baseline time-fixed variables); and (B) longitudinal model (with both time-fixed and time-varying variables, when appropriate). All variables were first tested in univariable models. Those with $\mathrm{p}<0.20$ were selected for multivariable analysis. In the final model we used forward selection and included variables that were significantly associated with the outcome $(\mathrm{p}<0.05)$, taking collinearity into account. Repeated observations of the outcome over time and observations stemming from the same country were not assumed to be independent. The exchangeable working correlation structure was used to handle correlation between repeated measures (first level), and country was added as a covariate in all models to adjust for this higher level of correlation (second level).

Sensitivity analyses. In addition to using DAS44 (main outcome, as per protocol), we repeated all analyses using Clinical Disease Activity Index remission $(\leq 2.8) / \mathrm{LDA}(\leq 10)$ and Simplified Disease Activity Index remission $(\leq 3.3) / \mathrm{LDA}(\leq 11)$ to define failure to apply T2T (similar to the main analysis, only outcome differs), each in separate models.

\section{RESULTS}

Baseline demographics and disease status. Complete baseline data were available on 571 patients (4427 visits) and 439 (76.9\%) had complete 2-year followup. Reasons for discontinuation were subject withdrew consent (52), subject lost to followup (25), major protocol violation(s) with study noncompliance (14), subject noncompliant with protocol (13), serious adverse event (10), other/unspecified (10), worsening of intercurrent medical condition (5), and investigator judgment (3). Complete details of patient baseline characteristics are described in the RA BIODAM study report ${ }^{14}$. Overall, the patient population comprised a demographically typical cohort of patients with RA, the majority being female (76\%) and with mean age of 55.7 years. Mean disease duration was 6.5 years and $52 \%$ had prior exposure to a csDMARD. Patients had active disease at baseline with a mean of 8.4 swollen joints, 13.6 tender joints, DAS28 of 5.2, DAS44 of 3.8, and CRP of $14.9 \mathrm{mg} / \mathrm{l}$. The majority (77.7\%) were either RF- or ACPA-positive.

Adherence to $T 2 T$. The percentage of patient visits in which there was failure to adhere to T2T was relatively stable over time. In total, there was failure of adherence to a T2T-REM strategy in 1765 (40.5\%) of visits, with the following reasons for this being provided in the electronic case report form (eCRF): physician decision - current treatment acceptable [534 (30.3\%)], physician decision - other [with specification; $93(5.3 \%)$ ], patient decision [with specification; $52(2.9 \%)$ ], other [40 (2.3\%)], physician decision - concern regarding adverse event [22 (1.2\%)], and unspecified [1024 (58.0\%)]. There was failure of adherence to a T2T-LDA strategy in $1098(25.2 \%)$ of visits, with the following reasons provided in the eCRF: physician decision - current treatment acceptable [519 (47.3\%)], physician decision - other [with specification; $93(8.5 \%)$ ], patient decision [with specification; 52 $(4.7 \%)]$, physician decision - concern regarding adverse event [22 (2.0\%)], other [38 (3.5\%)], and unspecified [374 $(34.1 \%)]$. The number of patients for whom there was failure to adhere to T2T for 1 or more visits is provided in Figure 1. Failure to adhere to T2T was observed for a majority of patient visits (i.e., $>50 \%$ of all visits) in 70 patients $(12.3 \%$ ) for T2T-REM and in 31 patients $(5.4 \%)$ for T2T-LDA.

At the first 3-month followup visit, failure of adherence to T2T-REM was evident in $46 \%$ of visits, and for T2T-LDA this was $33 \%$. Over the 2-year followup, there was a small decrease in the proportion of visits for which T2T-REM was not applied to $41 \%$, and a decrease for T2T-LDA to $20 \%$ of visits (Figure 2).

Main analysis: predictors of failure to implement T2T-REM. Older age and female sex were independent predictors of failure to implement T2T-REM in both the baseline and the longitudinal models (Table 1 and Table 2). Higher level of education predicted a lower likelihood of failure to implement T2-REM (longitudinal models only). Among disease severity factors, a higher HAQ at baseline (but not during followup) was associated with failure to implement T2T-REM, while ACPA positivity was associated with increased likelihood of implementing T2T-REM (only in the longitudinal models). For disease activity measures, a higher number of tender joints both at baseline and during followup was associated with a lower likelihood of implementing T2T-REM while the number of swollen joints had the opposite effect (only longitudinal model).

Secondary analysis: predictors of failure to implement T2T-LDA. In contrast to the main analysis of T2T-REM, older age and female sex were not associated with failure to

Personal non-commercial use only. The Journal of Rheumatology Copyright @ 2020 . All rights reserved. 


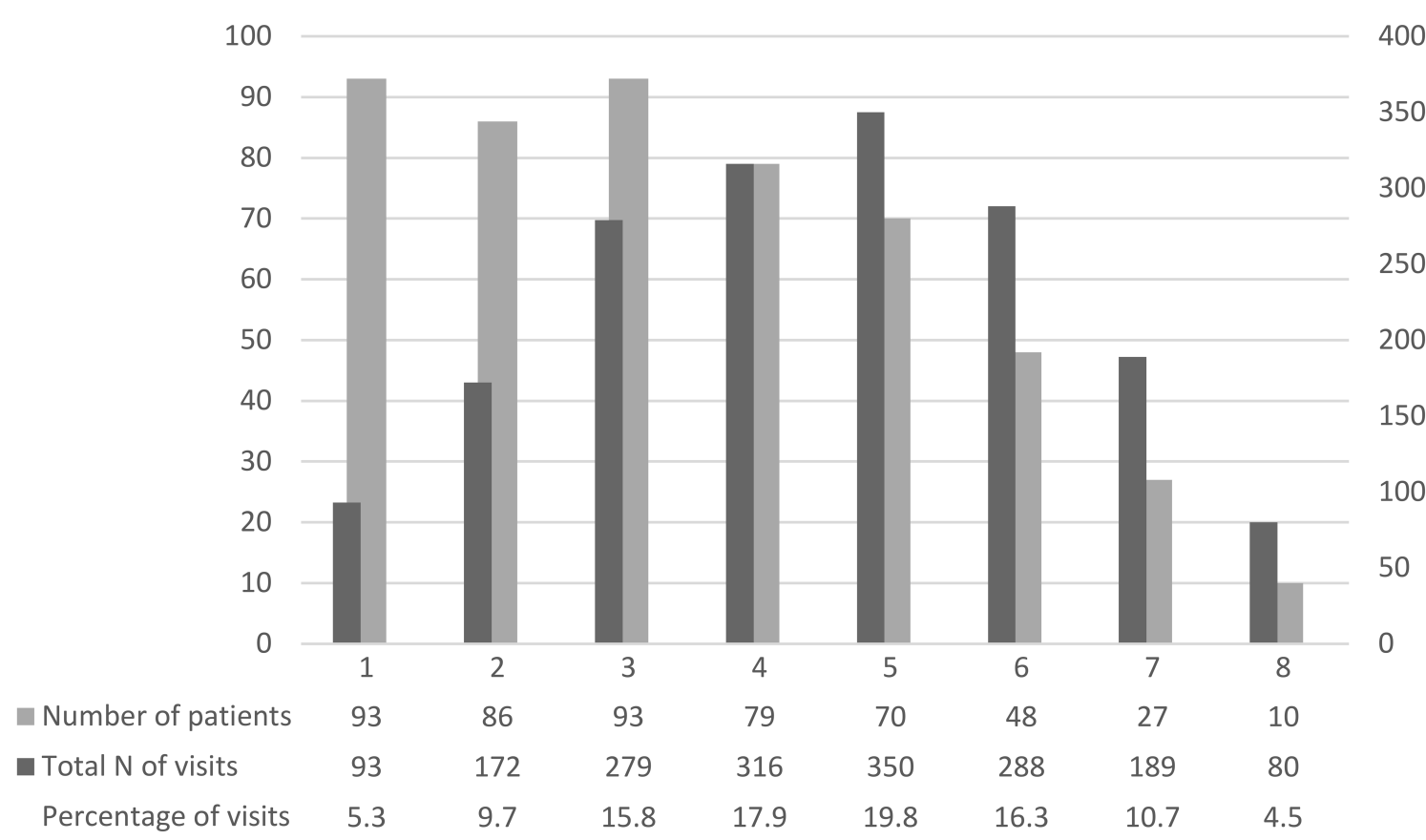

Number of patients

(B) T2T-LDA

Total number of visits

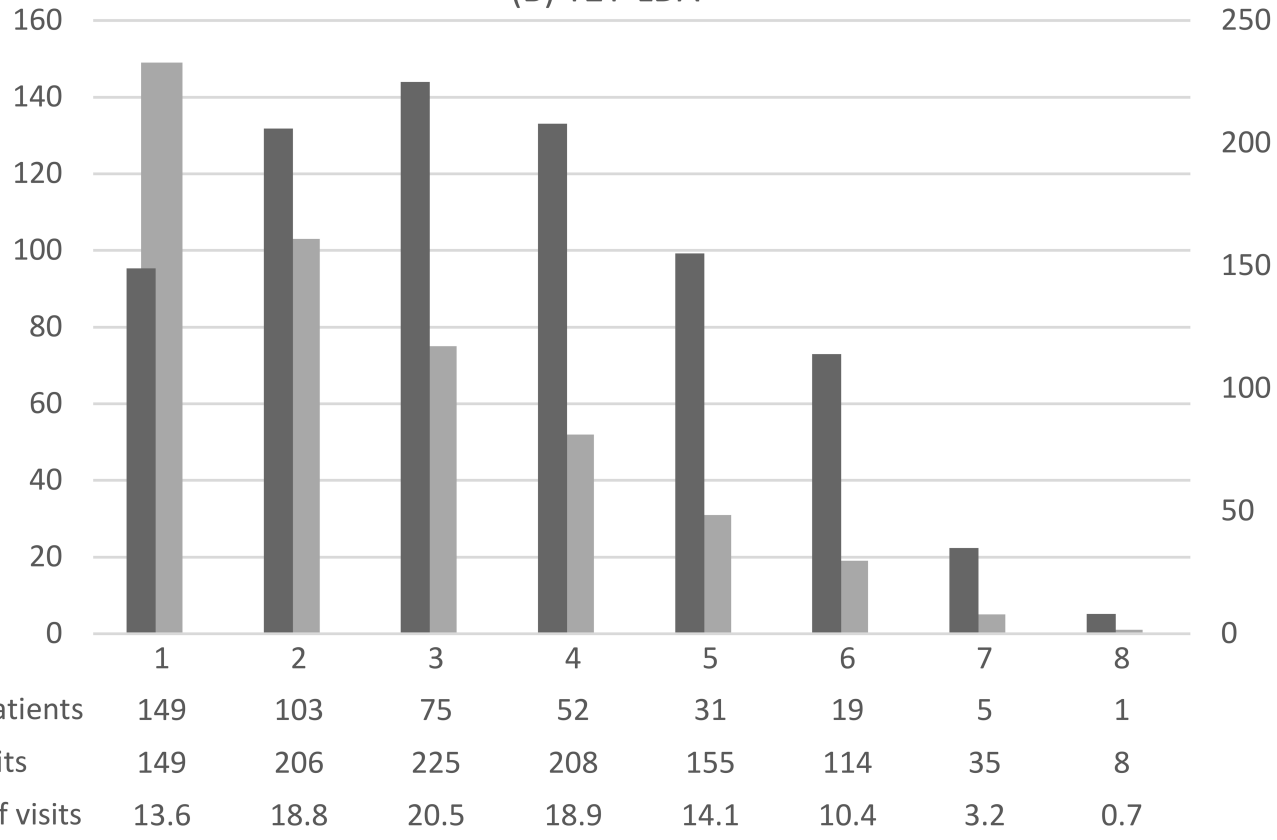

Figure 1. Failure of adherence to a T2T treatment strategy according to the number of visits per patient assessed every 3 months over 2 years in the RA BIODAM cohort. (A) T2T according to DAS44-REM. (B) T2T according to DAS44-LDA. Note: X-axis represents the number of visits where T2T was not applied; in Figure 1A, in 79 patients, rheumatologists failed to apply T2T-REM in 4 visits [total $79 \times$ $4=316$; which corresponds to $17.9 \%$ of all $(\mathrm{N}=1765)$ visits where T2T-REM was not applied]. T2T: treat-to-target; RA: rheumatoid arthritis; REM: remission; LDA: low disease activity; DAS44: 44-joint count Disease Activity Score.

implement T2T-LDA; level of education also played no role (Table 3 and Table 4). However, ACPA positivity was associated with increased likelihood of implementing
T2T-LDA as also noted for T2T-REM. In contrast to the analysis of T2T-REM, a higher number of comorbidities and smoking were independent predictors of failure to implement 


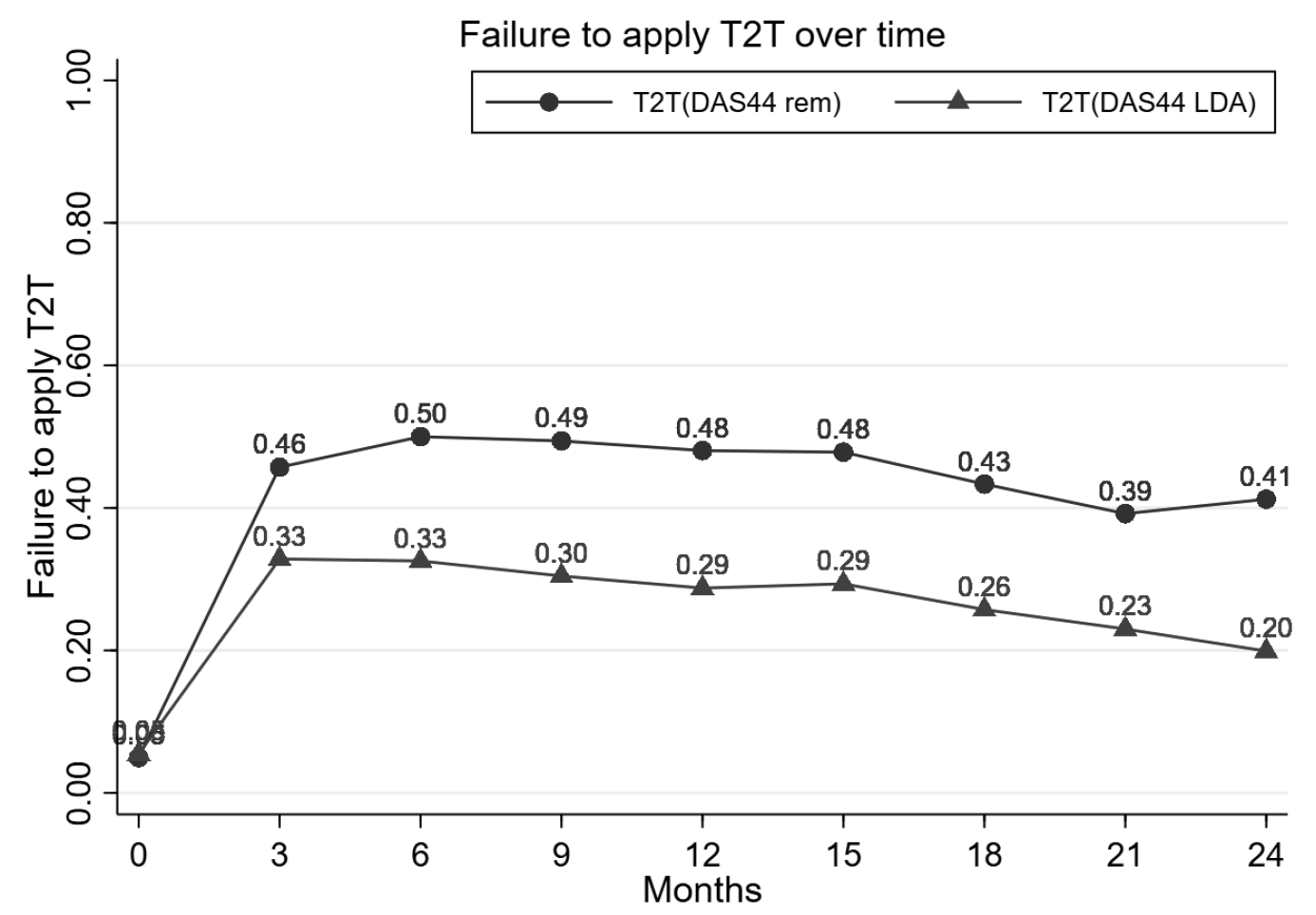

Figure 2. Proportion of visits in which T2T was not followed. In total, rheumatologists failed to appropriately apply the T2T-REM approach in 1765 visits (40.5\%) and the T2T-LDA approach in 1098 visits (25.2\%). T2T: treat-to-target; REM: remission; LDA: low disease activity; DAS44: 44-joint count Disease Activity Score.

T2T-LDA, while being in an academic center was associated with an increased likelihood of implementing T2T-LDA (only in longitudinal model). Higher TJC was associated with failure to implement T2T-LDA but there was no independent effect of SJC.

Sensitivity analyses. The results of all sensitivity analyses are similar to the ones from the main analysis and are shown in detail in Supplementary Tables 1 and 2 (available with the online version of this article).

\section{DISCUSSION}

Our data show that over the 2-year followup with visits every 3 months, there was a lack of implementation of the protocol-specified T2T treatment strategy in $40 \%$ of the visits, this being due mainly to physician decision that treatment was acceptable, among the specified reasons. There was a small decrease over 2 years in the proportion of visits for which implementation of treatment intensification aimed at T2T-REM was not applied, although for T2T-LDA the proportion gradually decreased from $33 \%$ to $20 \%$. Analysis of predictors of adherence to the T2T strategy suggests potential gaps in the care of female patients and smokers and demonstrates the value placed by rheumatologists on clinical biomarkers of prognosis to guide treatment decisions and their reluctance to escalate treatment in the absence of definitive physical findings of synovitis.

It is now widely accepted that attaining stringent clinical remission is associated with optimal outcomes in $\mathrm{RA}^{16,17,18,19,20,21}$. A major challenge for T2T is its implementation because of insufficient adherence and persistence, which leads to flares and increased disease activity ${ }^{10,22,23}$. Physicians raise concerns regarding the time expended on systematic joint assessments, possibility for adverse events, costs of therapy, and discordance between their own assessments of the level of disease activity, and the values provided by composite disease activity measures, particularly in the setting of damaged and tender joints and concomitant conditions such as osteoarthritis and fibromyalgia ${ }^{24,25,26}$. Consequently, LDA has also been proposed as a valuable alternative target, especially in established disease, because targeting LDA also leads to acceptable outcomes ${ }^{27}$.

A report described T2T protocol adherence in The BeST study (Dutch acronym for treatment strategies), a multicenter, randomized, clinical trial started in 2000 in the Netherlands, when T2T was not daily practice ${ }^{28}$. The aim was to evaluate the efficacy of 4 treatment strategies in 508 cases of early active RA defined according to the 1987 ACR criteria. The DAS was measured every 3 months, and this was used to inform treatment decisions targeted at LDA (DAS44 $\leq 2.4$ ) by the rheumatologist. Protocol adherence to a T2T strategy in BeST was compared with adherence to T2T in the IMPROVED study (acronym for Induction therapy with MTX and prednisone in RA or very early arthritic disease), which was also a multicenter, randomized, clinical trial

Personal non-commercial use only. The Journal of Rheumatology Copyright $\odot$ 2020. All rights reserved. 
Table 1. Baseline model: baseline predictors of failure to apply T2T-REM during 2-year followup in the RA BIODAM cohort.

\begin{tabular}{|c|c|c|c|}
\hline Variables & $\begin{array}{l}\text { Univariable OR } \\
\qquad \begin{array}{l}(95 \% \mathrm{CI}) \\
\mathrm{N}=544-571\end{array}\end{array}$ & Univariable $\mathrm{p}$ & $\begin{array}{l}\text { Multivariable OR } \\
\qquad \begin{array}{c}(95 \% \mathrm{CI}), \\
\mathrm{N}=549\end{array}\end{array}$ \\
\hline Age, yrs & $1.01(1.01-1.02)$ & $<0.001$ & $1.01(1.01-1.02)$ \\
\hline Sex, female & $1.36(1.11-1.66)$ & 0.003 & $1.35(1.11-1.64)$ \\
\hline Disease duration, yrs & $1.01(0.99-1.02)$ & 0.271 & $\dagger$ \\
\hline Education, yrs & $0.96(0.94-0.98)$ & 0.001 & $¥$ \\
\hline No. comorbidities & $1.11(1.04-1.19)$ & 0.003 & $¥$ \\
\hline Current smoker & $1.07(0.90-1.28)$ & 0.418 & $\dagger$ \\
\hline Type of center, academic & $1.01(0.85-1.20)$ & 0.935 & $\dagger$ \\
\hline RF positivity & $0.89(0.74-1.08)$ & 0.233 & $\dagger$ \\
\hline ACPA positivity & $0.81(0.68-0.97)$ & 0.024 & $¥$ \\
\hline RF and/or ACPA positivity & $0.85(0.69-1.05)$ & 0.136 & $¥$ \\
\hline $\operatorname{PtGA}(0-10)$ & $1.07(1.03-1.11)$ & $<0.001$ & $¥$ \\
\hline PGA $(0-10)$ & $1.02(0.98-1.07)$ & 0.351 & $\dagger$ \\
\hline Swollen joint count $(0-44)$ & $1.00(0.99-1.02)$ & 0.514 & $\dagger$ \\
\hline Tender joint count $(0-53)$ & $1.02(1.01-1.03)$ & $<0.001$ & $1.01(1.01-1.02)$ \\
\hline $\mathrm{ESR}, \mathrm{mm} / \mathrm{h}$ & $1.00(1.00-1.01)$ & 0.161 & $¥$ \\
\hline CRP, mg/l & $1.00(0.99-1.00)$ & 0.235 & $\dagger$ \\
\hline HAQ & $1.40(1.25-1.57)$ & $<0.001$ & $1.27(1.12-1.44)$ \\
\hline No. previous csDMARD & $1.07(1.00-1.15)$ & 0.057 & $¥$ \\
\hline Previous treatment with any csDMARD & $1.19(1.01-1.40)$ & 0.041 & $¥$ \\
\hline
\end{tabular}

$\dagger$ Not selected from the univariable analysis $(\mathrm{p}>0.20)$. $¥$ Not significant in the multivariable analysis $(\mathrm{p}>0.05)$. Country added as a covariate in all univariable models and in the final multivariable model. Final model also adjusted for type of treatment (csDMARD and/or bDMARD). Values in bold face are statistically significant. T2T: treat-to-target; REM: remission; RA: rheumatoid arthritis; RF: rheumatoid factor; ACPA: anticitrullinated protein antibody; PtGA: patient's global assessment; PGA: physician's global assessment; ESR: erythrocyte sedimentation rate; CRP: C-reactive protein; HAQ: Health Assessment Questionnaire; csDMARD: conventional synthetic disease-modifying antirheumatic drug; bDMARD: biological DMARD.

started in the Netherlands in 2007 and including some of the centers that participated in the BeST study ${ }^{27}$. The IMPROVED study recruited 479 early RA patients defined according to the $2010 \mathrm{ACR} / \mathrm{EULAR}$ classification criteria and 122 undifferentiated arthritis patients, who started induction therapy with MTX and tapered high-dose prednisone followed by treatment every 4 months targeted at DAS44-REM $(<1.6)$. Protocol adherence decreased over time in both studies, more so in the DAS $<1.6$-targeted study, and was $80 \%$ in BeST and 60\% in IMPROVED at 2 years of followup. In both studies, violations were associated with rheumatologists' disagreement with how the measured DAS represented actual disease activity, or with the next treatment step. These data are similar to what we report for protocol adherence in the RA BIODAM cohort. Following a protocol that aims at a stricter treatment target is more difficult because it may be perceived as conveying no additional clinical benefit and enhancing risks of side effects and/or higher costs. Rheumatologists have said that the measured DAS overestimates actual disease activity in a DAS $<1.6-$ steered treatment protocol compared to a DAS $\leq 2.4-$ steered treatment protocol ${ }^{29}$. The COBRA study also aimed treatment decisions to attain DAS-REM and showed comparable protocol violations during 6 months of followup
$(24 \%)^{30}$. In a 3-year retrospective followup of Australian patients with RA who were DMARD-naive, had disease duration of less than 1 year, and had treatment intensification according to DAS28 assessment, deviation from protocol occurred in $30.6 \%, 29.0 \%$, and $32.3 \%$ in the periods $6-12$, 12-24, and 24-36 months after treatment initiation, respectively ${ }^{31}$.

A significant difference of this past experience with T2T strategic decision making compared to the RA BIODAM cohort is that adherence to a treatment strategy based on T2T-LDA substantially improved over time, which could reflect a process of enhanced awareness to follow T2T, made possible by an alert mechanism built into the RA BIODAM eCRF platform. This simple tool could be integrated into an electronic medical record, suggesting a potential solution for enhancing best practices in the treatment of RA. It is also possible that greater flexibility and a greater number of options for treatment change in RA BIODAM promote adherence to a T2T strategy compared to the studies that were conducted before RA BIODAM.

In the longitudinal analysis of predictors of failure to implement a T2T strategy, differences were found when using a strategy based on targeting REM or LDA (both according to DAS44). A higher number of swollen joints during

Personal non-commercial use only. The Journal of Rheumatology Copyright $\subset$ 2020. All rights reserved 
Table 2. Longitudinal model: time-fixed and time-varying predictors of failure to apply T2T-REM during 2-year followup in the RA BIODAM cohort.

\begin{tabular}{|c|c|c|c|}
\hline Variables & $\begin{array}{c}\text { Univariable OR } \\
(95 \% \mathrm{CI}), \mathrm{N}=544-571\end{array}$ & Univariable $\mathrm{p}$ & $\begin{array}{l}\text { Multivariable OR } \\
(95 \% \mathrm{CI}), \mathrm{N}=545\end{array}$ \\
\hline Age, yrs & $1.01(1.01-1.02)$ & $<0.001$ & $1.01(1.00-1.02)$ \\
\hline Sex, female & $1.36(1.11-1.66)$ & 0.003 & 1.43 (1.15-1.77) \\
\hline Disease duration, yrs & $1.01(0.99-1.02)$ & 0.271 & $\dagger$ \\
\hline Education, yrs & $0.96(0.94-0.98)$ & 0.001 & $0.97(0.94-0.99)$ \\
\hline No. comorbidities & $1.11(1.04-1.19)$ & 0.003 & $¥$ \\
\hline Current smoker & $1.07(0.90-1.28)$ & 0.418 & $\dagger$ \\
\hline Type of center, academic & $1.01(0.85-1.20)$ & 0.935 & $\dagger$ \\
\hline RF positivity & $0.89(0.74-1.08)$ & 0.233 & $\dagger$ \\
\hline ACPA positivity & $0.81(0.68-0.97)$ & 0.024 & $0.79(0.65-0.95)$ \\
\hline $\mathrm{RF}$ and/or ACPA positivity & $0.85(0.69-1.05)$ & 0.136 & $¥$ \\
\hline $\operatorname{PtGA}(0-10) \ddagger$ & $1.00(0.97-1.03)$ & 0.966 & $\dagger$ \\
\hline PGA $(0-10)^{\ddagger}$ & $0.91(0.89-0.94)$ & $<0.001$ & $¥$ \\
\hline Swollen joint count $(0-44) \ddagger$ & $0.93(0.91-0.95)$ & $<0.001$ & $0.92(0.90-0.94)$ \\
\hline Tender joint count $(0-53) \ddagger$ & $0.99(0.98-1.00)$ & 0.002 & $1.02(1.00-1.03)$ \\
\hline $\mathrm{ESR}, \mathrm{mm} / \mathrm{h}^{\ddagger}$ & $1.00(1.00-1.00)$ & 0.878 & $\dagger$ \\
\hline $\mathrm{CRP}, \mathrm{mg} / \mathrm{l}^{\ddagger}$ & $0.99(0.98-0.99)$ & 0.003 & $¥$ \\
\hline $\mathrm{HAQ}^{\dagger}$ & $1.03(0.91-1.15)$ & 0.681 & $\dagger$ \\
\hline No. previous csDMARD & $1.07(1.00-1.15)$ & 0.057 & $¥$ \\
\hline Previous treatment with any csDMARD & $1.19(1.01-1.40)$ & 0.041 & $¥$ \\
\hline
\end{tabular}

$\dagger$ Not selected from the univariable analysis ( $\mathrm{p}>0.20)$. $¥$ Not significant in the multivariable analysis $(\mathrm{p}>0.05)$.

$\doteqdot$ Modeled as time-varying. Country added as a covariate in all univariable models and in the final multivariable model. Final model also adjusted for type of treatment (csDMARD and/or bDMARD). Values in bold face are statistically significant. T2T: treat-to-target; REM: remission; RA: rheumatoid arthritis; RF: rheumatoid factor; ACPA: anticitrullinated protein antibody; PtGA: patient's global assessment; PGA: physician's global assessment; ESR: erythrocyte sedimentation rate; CRP: C-reactive protein; HAQ: Health Assessment Questionnaire; csDMARD: conventional synthetic disease-modifying antirheumatic drug; bDMARD: biological DMARD.

followup were associated with increased implementation of T2T-REM, while a higher number of tender joints had the opposite effect, both at baseline and during followup. This finding supports the view that treatment targeted to remission is strongly driven by the importance attached by rheumatologists to eliminating inflammation in swollen joints. The higher the score of this objective sign of inflammation (irrespective of CRP/ESR), the higher the likelihood that rheumatologists will decide on more "aggressive" treatment strategies. Being ACPA-positive was also associated with a greater likelihood that the T2T strategy would be implemented. Rheumatologists might feel more confident in implementing strict treatment strategies in cases where they also have more confidence in the diagnosis. In addition, ACPA positivity is a known adverse prognostic factor, so rheumatologists appear to take into account prognostic factors when deciding whether to follow T2T, and follow it more in the presence of ACPA positivity. A limitation of the anticyclic citrullinated peptide antibody test is that it is relatively invariant to change over time, so it is not assessed in a prospective manner. Additional prognostic biomarkers that are modifiable and could be targeted for treatment intervention therefore represent a major unmet need in the optimal management of RA. This unmet need reinforces the rationale for the development of the RA BIODAM cohort because it may ultimately enhance the adoption of T2T strategies in real-world practice.

Several demographic variables influenced implementation of T2T. In particular, we found that older age and female sex were associated with a lower likelihood of implementing T2T-REM both in models with only time-fixed variables (baseline model) and in longitudinal models also incorporating time-varying variables. But interestingly, this association was not found when modeling T2T-LDA. Potential reasons for lack of implementation of T2T toward a target of remission in older individuals could be concern regarding adverse events, difficulty in distinguishing inflammatory from degenerative joint pain, and comorbidities that result in a more conservative approach to therapy. The less intensive treatment approach adopted in women is disconcerting and requires further study as to causal factors and its effect on attainment of remission and prevention of joint damage. Higher level of education was also found to be associated with higher likelihood of implementation of T2T-REM (but not T2T-LDA). More educated patients may be more proactive in finding information about optimal management of RA. Heightened awareness of the effect of inflammation on joint damage and even information about T2T strategies as the optimal mode of treatment may persuade patients to accept treatment intensification. A higher number of comor-

Personal non-commercial use only. The Journal of Rheumatology Copyright @ 2020 . All rights reserved. 
Table 3. Baseline model: baseline predictors of failure to apply T2T-LDA during 2-year followup of the RA BIODAM cohort.

\begin{tabular}{|c|c|c|c|}
\hline Variables & $\begin{array}{c}\text { Univariable OR } \\
(95 \% \mathrm{CI}), \mathrm{N}=544-571\end{array}$ & Univariable $\mathrm{p}$ & $\begin{array}{c}\text { Multivariable OR } \\
(95 \% \mathrm{CI}), \mathrm{N}=549\end{array}$ \\
\hline Age, yrs & $1.01(1.00-1.01)$ & 0.096 & $¥$ \\
\hline Sex, female & $1.01(0.81-1.25)$ & 0.963 & $\dagger$ \\
\hline Disease duration, yrs & $1.00(0.99-1.02)$ & 0.528 & $\dagger$ \\
\hline Education, yrs & $0.97(0.95-1.00)$ & 0.032 & $¥$ \\
\hline No. comorbidities & $1.17(1.08-1.26)$ & $<0.001$ & $1.10(1.02-1.19)$ \\
\hline Current smoker & $1.24(1.01-1.53)$ & 0.041 & $1.32(1.08-1.63)$ \\
\hline Type of center, academic & $0.82(0.67-1.00)$ & 0.050 & $0.81(0.66-0.99)$ \\
\hline RF positivity & $0.63(0.51-0.77)$ & $<0.001$ & $£$ \\
\hline ACPA positivity & $0.57(0.47-0.70)$ & $<0.001$ & $£$ \\
\hline RF and/or ACPA positivity & $0.58(0.46-0.73)$ & $<0.001$ & $0.63(0.50-0.80)$ \\
\hline DAS44-ESR LDA & $1.06(0.58-1.96)$ & 0.845 & $\dagger$ \\
\hline $\operatorname{PtGA}(0-10)$ & $1.07(1.03-1.12)$ & 0.001 & $¥$ \\
\hline PGA $(0-10)$ & $1.07(1.02-1.13)$ & 0.009 & $¥$ \\
\hline Swollen joint count $(0-44)$ & $1.01(1.00-1.03)$ & 0.075 & $¥$ \\
\hline Tender joint count $(0-53)$ & $1.03(1.03-1.04)$ & $<0.001$ & $1.03(1.02-1.04)$ \\
\hline $\mathrm{ESR}, \mathrm{mm} / \mathrm{h}$ & $1.00(1.00-1.01)$ & 0.516 & $\dagger$ \\
\hline CRP, mg/l & $1.00(0.99-1.00)$ & 0.908 & $\dagger$ \\
\hline HAQ & $1.29(1.12-1.49)$ & $<0.001$ & $¥$ \\
\hline No. previous csDMARD & $1.02(0.94-1.11)$ & 0.622 & $\dagger$ \\
\hline Previous treatment with any csDMARD & $1.10(0.91-1.33)$ & 0.318 & $\dagger$ \\
\hline
\end{tabular}

$\dagger$ Not selected from the univariable analysis $(\mathrm{p}>0.20)$. ¥ Not significant in the multivariable analysis $(\mathrm{p}>0.05)$. $£:$ RF or ACPA positivity entered into multivariate model. Country added as a covariate in all univariable models and in the final multivariable model. Final model also adjusted for type of treatment (csDMARD and/or bDMARD). Values in bold face are statistically significant. T2T: treat-to-target; LDA: low disease activity; RA: rheumatoid arthritis; RF: rheumatoid factor; ACPA: anticitrullinated protein antibody; DAS44-ESR: 44-joint count Disease Activity Score based on erythrocyte sedimentation rate; PtGA: patient's global assessment; PGA: physician's global assessment; CRP: C-reactive protein; HAQ: Health Assessment Questionnaire; csDMARD: conventional synthetic disease-modifying antirheumatic drug; bDMARD: biological DMARD.

bidities and being a current smoker were independently associated with higher likelihood to fail to apply T2T-LDA (not T2T-REM). Rheumatologists may be concerned about adverse events and interaction with other therapies in such patients, which could lead them to be more conservative in implementing aggressive treatment strategies. The association between higher HAQ (more disability) and higher likelihood to fail T2T was also seen in the baseline model for T2T-REM. Previous reports of factors accounting for protocol deviations in $\mathrm{T} 2 \mathrm{~T}$ studies have similarly cited comorbidity, drug toxicity, and patient-reported factors such as helplessness, modified HAQ, pain, and fatigue $32,33,34,35,36,37$. Body mass index, baseline DAS28, and TJC were also associated with the number of deviations.

The outcomes used in this analysis incorporate treatment decisions, and thus reflect the rheumatologists' perceptions about T2T. We have observed that rheumatologists in the RA BIODAM study went outside protocol and made decisions in each case based on their best knowledge. On one hand, this informs us (or better, confirms) that confounding by indication is present in RA BIODAM, but on the other hand, it also indicates that this cohort truly reflects how patients with RA are treated in daily clinical practice. Keeping this in mind, this analysis sheds light into what are perceived by rheumatologists as facilitators and barriers when deciding to apply T2T in clinical practice. Having a high number of swollen joints, being ACPA-positive, and being more educated were found to make the application of T2T more likely. On the contrary, older age, female sex, a high number of tender joints, high baseline HAQ, a high number of comorbidities, and being a smoker were perceived by rheumatologists as barriers to implementation of T2T.

Despite a protocol-specific requirement to adhere to a T2T treatment strategy, this was not implemented in $40 \%$ of patient visits for the DAS44-REM target and in $25 \%$ for the DAS44-LDA target, although there was a steady decline in DAS44-LDA failures over the 2-year followup. Rheumatologists are influenced by objective measures such as swollen joints and positivity for ACPA in deciding on implementation of treatment intensification because of their association with prognosis. The validation of modifiable biomarkers of RA prognosis using the resources generated in RA BIODAM may therefore provide rheumatologists with additional tools that will ease decision making in clinical practice. 
Table 4. Longitudinal model: time-fixed and time-varying predictors of failure to apply T2T-LDA during 2-year followup of the RA BIODAM cohort.

\begin{tabular}{|c|c|c|c|}
\hline Variables & $\begin{array}{c}\text { Univariable OR } \\
(95 \% \mathrm{CI}), \mathrm{N}=544-571\end{array}$ & Univariable $\mathrm{p}$ & $\begin{array}{c}\text { Multivariable OR } \\
(95 \% \mathrm{CI}), \mathrm{N}=554\end{array}$ \\
\hline Age, yrs & $1.01(1.00-1.01)$ & 0.096 & $¥$ \\
\hline Sex, female & $1.01(0.81-1.25)$ & 0.963 & $\dagger$ \\
\hline Disease duration, yrs & $1.00(0.99-1.02)$ & 0.528 & $\dagger$ \\
\hline Education, yrs & $0.97(0.95-1.00)$ & 0.032 & $¥$ \\
\hline No. comorbidities & $1.17(1.08-1.26)$ & $<0.001$ & $1.11(1.03-1.20)$ \\
\hline Current smoker & $1.24(1.01-1.53)$ & 0.041 & $1.26(1.03-1.53)$ \\
\hline Type of center, academic & $0.82(0.67-1.00)$ & 0.050 & $0.81(0.68-0.98)$ \\
\hline RF positivity & $0.63(0.51-0.77)$ & $<0.001$ & $*$ \\
\hline ACPA positivity & $0.57(0.47-0.70)$ & $<0.001$ & $*$ \\
\hline $\mathrm{RF}$ and/or ACPA positivity & $0.58(0.46-0.73)$ & $<0.001$ & $0.66(0.53-0.82)$ \\
\hline $\operatorname{PtGA}(0-10)^{\ddagger}$ & $1.11(1.08-1.14)$ & $<0.001$ & $¥$ \\
\hline $\operatorname{PGA}(0-10)^{\ddagger}$ & $1.06(1.03-1.10)$ & $<0.001$ & $¥$ \\
\hline Swollen joint count $(0-44) \ddagger$ & $1.00(0.98-1.02)$ & 0.962 & $\dagger$ \\
\hline Tender joint count $(0-53) \ddagger$ & $1.03(1.03-1.04)$ & $<0.001$ & $1.02(1.01-1.03)$ \\
\hline $\mathrm{ESR}, \mathrm{mm} / \mathrm{h}$ & $1.01(1.00-1.01)$ & 0.013 & $¥$ \\
\hline $\mathrm{CRP}, \mathrm{mg} / \mathrm{l}^{\ddagger}$ & $1.00(0.99-1.00)$ & 0.453 & $\dagger$ \\
\hline $\mathrm{HAQ}^{\ddagger}$ & $1.43(1.26-1.61)$ & $<0.001$ & $¥$ \\
\hline No. previous csDMARD & $1.02(0.94-1.11)$ & 0.622 & $\dagger$ \\
\hline Previous treatment with any csDMARD & $1.10(0.91-1.33)$ & 0.318 & $\dagger$ \\
\hline
\end{tabular}

$\dagger$ Not selected from the univariable analysis $(\mathrm{p}>0.20)$. $¥$ Not significant in the multivariable analysis $(\mathrm{p}>0.05)$. $¥$ Modeled as time-varying. $*$ RF or ACPA positivity entered into multivariate model. Country added as a covariate in all univariable models and in the final multivariable model. Final model also adjusted for type of treatment (csDMARD and/or bDMARD). Values in bold face are statistically significant. T2T: treat-to-target; LDA: low disease activity; RA: rheumatoid arthritis; RF: rheumatoid factor; ACPA: anticitrullinated protein antibody; SKATe6055PtGA: patient's global assessment; PGA: physician's global assessment; ESR: erythrocyte sedimentation rate; CRP: C-reactive protein; HAQ: Health Assessment Questionnaire; csDMARD: conventional synthetic disease-modifying antirheumatic drug; bDMARD: biological DMARD.

\section{ACKNOWLEDGMENT}

The authors acknowledge the contribution of the following RA BIODAM investigators and their local site teams: Thomas Neumann, Department of Internal Medicine III/Rheumatology/Osteology, Universitätsklinikum der Friedrich-Schiller-Universität Jena, Jena, Germany; Renée Allaart, Department of Rheumatology, Leiden University Medical Centre, Leiden, the Netherlands; Christopher Ritchlin, Division of Allergy, Immunology, and Rheumatology, University of Rochester Medical Center, Rochester, New York, USA; Joan Bathon, Division of Rheumatology, Columbia University Medical Center and Columbia University College of Physicians and Surgeons, New York, New York, USA; René-Marc Flipo, Centre d'Investigation Clinique, CHRU de Lille, Lille, France; Proton Rahman, Memorial University, St. John's, Newfoundland and Labrador, Canada; Carol Hitchon, Arthritis Center, Department of Internal Medicine, Rheumatology Section, University of Manitoba, Winnipeg, Manitoba, Canada; Wolfgang Spieler, Osteology and Rheumatology, ZeFOR GmbH Center for Research, Zerbst, Germany; Leonardo Punzi, Department of Medicine, Università di Padova, Padua, Italy; Ingo Tarner, Department of Rheumatology and Clinical Immunology, Kerckhoff-Klinik, Bad Nauheim, Germany; Vivian Bykerk, Inflammatory Arthritis Center, Hospital for Special Surgery, New York, New York, USA; Philip J. Mease, Seattle Rheumatology Associates, Seattle, Washington, USA.

\section{DATA SHARING POLICY}

The datasets generated during and/or analyzed during the current study are available from the corresponding author on reasonable request.

Three different databases were developed by the coordinating project management group CaRE Arthritis Ltd., which were linked by the patient study ID:
1. Clinical database: clinical data was recorded in the RA BIODAM eCRF, and an interactive system of study queries was used to proactively verify data entry and address missing data within prespecified time frames.

2. Biosample biorepository: aliquoted sera, urine, and RNA biosamples were barcoded and stored at $-70^{\circ} \mathrm{C}$.

3. Imaging repository: all anonymized Digital Imaging and Communications in Medicine radiographs of hands and feet passed quality assurance procedures.

Access to all RA BIODAM data and biosamples will be made available to academic and not-for-profit entities. This will require the submission of a study proposal to the scientific committee; see www.carearthritis.com.

\section{ONLINE SUPPLEMENT}

Supplementary material accompanies the online version of this article.

\section{REFERENCES}

1. Stoffer MA, Schoels MM, Smolen JS, Aletaha D, Breedveld FC, Burmester G, et al. Evidence for treating rheumatoid arthritis to target: results of a systematic literature search update. Ann Rheum Dis 2016;75:16-22.

2. Schipper LG, Vermeer M, Kuper HH, Hoekstra MO, Haagsma CJ, Den Broeder AA, et al. A tight control treatment strategy aiming for remission in early rheumatoid arthritis is more effective than usual care treatment in daily clinical practice: a study of two cohorts in the Dutch Rheumatoid Arthritis Monitoring registry. Ann Rheum Dis 2012;71:845-50.

3. Schipper LG, van Hulst LT, Grol R, van Riel PL, Hulscher ME, Fransen J. Meta-analysis of tight control strategies in rheumatoid arthritis: protocolized treatment has additional value with respect to the clinical outcome. Rheumatology 2010;49:2154-64.

Personal non-commercial use only. The Journal of Rheumatology Copyright @ 2020 . All rights reserved. 
4. Pope JE, Haraoui B, Rampakakis E, Psaradellis E, Thorne C, Sampalis JS, et al; Optimization of Adalimumab Trial Investigators. Treating to a target in established active rheumatoid arthritis patients receiving a tumor necrosis factor inhibitor: results from a real-world cluster-randomized adalimumab trial. Arthritis Care Res 2013;65:1401-9.

5. Vermeer M, Kievit W, Kuper HH, Braakman-Jansen LM, Bernelot Moens HJ, Zijlstra TR, et al. Treating to the target of remission in early rheumatoid arthritis is cost-effective: results of the DREAM registry. BMC Musculoskelet Disord 2013;14:350.

6. Provan SA, Semb AG, Hisdal J, Stranden E, Agewall S, Dagfinrud $\mathrm{H}$, et al. Remission is the goal for cardiovascular risk management in patients with rheumatoid arthritis: a cross-sectional comparative study. Ann Rheum Dis 2011;70:812-7.

7. Jurgens MS, Welsing PM, Geenen R, Bakker MF, Schenk Y, de Man YA, et al. The separate impact of tight control schemes and disease activity on quality of life in patients with early rheumatoid arthritis: results from the CAMERA trials. Clin Exp Rheumatol 2014;32:369-76.

8. Smolen JS, Landewe R, Breedveld FC, Buch M, Burmester G, Dougados M, et al. EULAR recommendations for the management of rheumatoid arthritis with synthetic and biological disease-modifying antirheumatic drugs: 2013 update. Ann Rheum Dis 2014;73:492-509.

9. Singh JA, Saag KG, Bridges SL Jr, Akl EA, Bannuru RR, Sullivan MC, et al. 2015 American College of Rheumatology guideline for the treatment of rheumatoid arthritis. Arthritis Rheumatol 2016;68:1-26.

10. Kuusalo L, Puolakka K, Kautiainen H, Blafield H, Eklund KK, Ilva $\mathrm{K}$, et al; Neo-RACo Study Group. Impact of physicians' adherence to treat-to-target strategy on outcomes in early rheumatoid arthritis in the NEO-RACo trial. Scand J Rheumatol 2015;44:449-55.

11. Maksymowych WP, Landewe R, Tak PP, Ritchlin CJ, Ostergaard M, Mease PJ, et al. Reappraisal of OMERACT 8 draft validation criteria for a soluble biomarker reflecting structural damage endpoints in rheumatoid arthritis, psoriatic arthritis, and spondyloarthritis: The OMERACT 9 v2 criteria. J Rheumatol 2009;36:1785-91.

12. Maksymowych WP, Fitzgerald O, Wells GA, Gladman DD, Landewe R, Ostergaard M, et al. Proposal for levels of evidence schema for validation of a soluble biomarker reflecting damage endpoints in rheumatoid arthritis, psoriatic arthritis, and ankylosing spondylitis, and recommendations for study design. J Rheumatol 2009;36:1792-9.

13. Aletaha D, Neogi T, Silman AJ, Funovits J, Felson DT, Bingham CO 3rd, et al. 2010 Rheumatoid Arthritis Classification Criteria: an American College of Rheumatology/European League Against Rheumatism collaborative initiative. Arthritis Rheum 2010;62:2569-81.

14. Maksymowych WP, FitzGerald O, Østergaard M, Homik J, van der Heijde D, Lambert RG, et al. The international RA BIODAM cohort for validation of soluble biomarkers in rheumatoid arthritis: cohort description. J Rheumatol 2020 Feb 15 (E-pub ahead of print).

15. Smolen JS, Aletaha D, Bijlsma JWJ, Breedveld FC, Boumpas D, Burmester G, et al; T2T Expert Committee. Treating rheumatoid arthritis to target: recommendations of an international task force. Ann Rheum Dis 2010;69:631-7.

16. Felson DT, Smolen JS, Wells G, Zhang B, van Tuyl LH, Funovits J, et al. American College of Rheumatology/European League Against Rheumatism provisional definition of remission in rheumatoid arthritis for clinical trials. Ann Rheum Dis 2011;70:404-13.

17. Kavanaugh A, Fleischmann RM, Emery P, Kupper H, Redden L, Guerette B, et al. Clinical, functional and radiographic consequences of achieving stable low disease activity and remission with adalimumab plus methotrexate or methotrexate alone in early rheumatoid arthritis: 26-week results from the randomised, controlled OPTIMA study. Ann Rheum Dis 2013;72:64-71.

18. Sakellariou G, Scire CA, Verstappen SM, Montecucco C, Caporali $\mathrm{R}$. In patients with early rheumatoid arthritis, the new ACR/EULAR definition of remission identifies patients with persistent absence of functional disability and suppression of ultrasonographic synovitis. Ann Rheum Dis 2013;72:245-9.

19. Thiele K, Huscher D, Bischoff S, Spathling-Mestekemper S, Backhaus M, Aringer M, et al; German Collaborative Arthritis Centres. Performance of the 2011 ACR/EULAR preliminary remission criteria compared with DAS28 remission in unselected patients with rheumatoid arthritis. Ann Rheum Dis 2013;72:1194-9.

20. Radner H, Smolen JS, Aletaha D. Remission in rheumatoid arthritis: benefit over low disease activity in patient reported outcomes and costs. Arthritis Res Ther 2014;16:R56.

21. Smolen JS, Breedveld FC, Burmester GR, Bykerk V, Dougados M, Emery P, et al. Treating rheumatoid arthritis to target: 2014 update of the recommendations of an international task force. Ann Rheum Dis 2016;75:3-15.

22. Pascual-Ramos V, Contreras-Yanez I, Villa AR, Cabiedes J, Rull-Gabayet M. Medication persistence over 2 years of follow-up in a cohort of early rheumatoid arthritis patients: associated factors and relationship with disease activity and with disability. Arthritis Res Ther 2009;11:R26.

23. Contreras-Yáñez I, Pascual-Ramos V. Window of opportunity to achieve major outcomes in early rheumatoid arthritis patients: how persistence with therapy matters. Arthritis Res Ther 2015;17:177.

24. Haraoui B, Smolen JS, Aletaha D, Breedveld FC, Burmester G, Codreanu C, et al; Treat to Target Taskforce. Treating rheumatoid arthritis to target: multinational recommendations assessment questionnaire. Ann Rheum Dis 2011;70:1999-2002.

25. Markusse IM, Dirven L, Han KH, van Groenendael JH, Ronday HK, Kerstens PJ, et al. Evaluating adherence to a treat-to-target protocol in recent-onset rheumatoid arthritis: reasons for compliance and hesitation. Arthritis Care Res 2015;68:446-53.

26. Bakker MF, Jacobs JW, Verstappen SM, Bijlsma JW. Tight control in the treatment of rheumatoid arthritis: efficacy and feasibility. Ann Rheum Dis 2007;66 Suppl 3:iii56-60.

27. Bergstra SA, Olivas O, Akdemir G, Riyazi N, Collée G, van Groenendae JH, et al. Further treatment intensification in undifferentiated and rheumatoid arthritis patients already in low disease activity has limited benefit towards physical functioning Arthritis Res Ther 2017;19:220.

28. Akdemir G, Markusse IM, Goekoop-Ruiterman YP, Steup-Beekman GM, Grillet BA, Kerstens PJ, et al. Rheumatologists' adherence to a disease activity score steered treatment protocol in early arthritis patients is less if the target is remission. Clin Rheumatol 2017;36:317-26.

29. Wolfe F, Michaud K, Pincus T, Furst D, Keystone E. The disease activity score is not suitable as the sole criterion for initiation and evaluation of anti-tumor necrosis factor therapy in the clinic: discordance between assessment measures and limitations in questionnaire use for regulatory purposes. Arthritis Rheum 2005;52:3873-9.

30. den Uyl D, ter Wee M, Boers M, Kerstens P, Voskuyl A, Nurmohamed $\mathrm{M}$, et al. A non-inferiority trial of an attenuated combination strategy ('COBRA-light') compared to the original COBRA strategy: clinical results after 26 weeks. Ann Rheum Dis 2014;73:1071-8.

31. Wabe N, Sorich MJ, Wechalekar MD, Cleland LG, McWilliams L, Lee A, et al. Characterising deviation from treat-to-target strategies for early rheumatoid arthritis: the first three years. Arthritis Res Ther 2015;17:48

32. Tymms K, Zochling J, Scott J, Bird P, Burnet S, de Jager J, et al. Barriers to optimal disease control for rheumatoid arthritis patients 
with moderate and high disease activity. Arthritis Care Res 2014;66:190-6.

33. Vermeer M, Kuper HH, Bernelot Moens HJ, Hoekstra M, Posthumus MD, van Riel PL, et al. Adherence to a treat-to-target strategy in early rheumatoid arthritis: results of the DREAM remission induction cohort. Arthritis Res Ther 2012;14:R254.

34. Wolfe F, Michaud K. Resistance of rheumatoid arthritis patients to changing therapy: discordance between disease activity and patients' treatment choices. Arthritis Rheum 2007;56:2135-42.

35. Barton JL, Imboden J, Graf J, Glidden D, Yelin EH, Schillinger D.
Patient-physician discordance in assessments of global disease severity in rheumatoid arthritis. Arthritis Care Res 2010;62:857-64.

36. Nicolau G, Yogui MM, Vallochi TL, Gianini RJ, Laurindo IM, Novaes GS. Sources of discrepancy in patient and physician global assessments of rheumatoid arthritis disease activity. J Rheumatol 2004;31:1293-6.

37. Pincus T, Esther R, DeWalt DA, Callahan LF. Social condition and self-management are more powerful determinants of health than access to care. Ann Intern Med 1998;129:406-11. 